Geopolítica(s) Revista de estudios sobre espacio y poder ISSN: 2172-3958

\title{
La política exterior de la Unión Europea en el Cáucaso y Asia Central: los límites de la apertura hacia el Este ${ }^{1}$
}

\author{
Carles Jovaní Gil ${ }^{2}$
}

Recibido: 18 de enero de 2016 / Aceptado: 20 de abril de 2016

Resumen. El presente artículo se propone estudiar la evolución de la política exterior de la Unión Europea en el Cáucaso meridional y Asia Central durante la Posguerra Fría. Con ello se pretende evaluar cuáles son sus principales intereses y limitaciones en la región, qué estrategias ha implementado en los últimos años, y en qué medida ha sido capaz de socavar el dominio de sus hegemones tradicionales. Como se pondrá de manifiesto, las debilidades crónicas comunitarias, las reticencias locales a ceder soberanía y la creciente competitividad geopolítica internacional comprometen cualquier aspiración europea de consagrarse en un horizonte próximo como un actor preeminente en el corazón del continente euroasiático.

Palabras clave: Unión Europea; Cáucaso; Asia Central; política exterior; geopolítica.

\section{[en] The European Union Foreign Policy in the Caucasus and Central Asia: The Limitations of the Opening towards the East}

\begin{abstract}
This article intends to study the evolution of the European Union foreign policy in the Southern Caucasus and Central Area throughout the Post-Cold War era. The aim is to analyze Brussels' fundamental interests and limitations in the area, the strategies it has implemented in the last few years, and the extent to which the EU has been able to undermine the regional hegemons' traditional supremacy. As will be highlighted, the Community's chronic weaknesses, the local determination to preserve sovereignty and an increasing international geopolitical competition undermine any European aspiration to become a pre-eminent actor at the heart of the Eurasian continent in the near future.
\end{abstract}

Keywords: European Union; Caucasus; Central Asia; foreign policy; geopolitics.

\section{[pt] A política externa da União Europeia na região do Cáucaso e da Ásia} Central: os limites da abertura a Leste

Resumo. O presente artigo propõe estudar a evolução da política externa da União Europeia no Sul do Cáucaso e da Ásia Central durante a Guerra Fria. Este é destinado a avaliar quais são os seus

1 El presente artículo se ha elaborado en el marco de una Beca Fundación Ramón Areces para Estudios de Postgrado en Ciencias Sociales.

2 EU External Relations and Diplomacy Studies Department, College of Europe, Verversdijk Campus, building E, ground floor - Brujas (Bélgica)

E-mail: carles.jovani@coleurope.eu 
principais interesses e limitações na região, quais as estratégias implementadas ao longo dos últimos anos e em que medida tem sido capaz de minar o domínio dos seus tradicionais hegemons. O presente artigo propõe estudar a evolução da política externa da União Europeia no Sul do Cáucaso e da Ásia Central durante a Guerra Fria. Estas disposições destinam-se a revelar os principais interesses e limitações de Bruxelas, analisar as estratégias implementadas nos últimos anos e para avaliar a medida em que a UE será capaz de contestar a hegemonia para os seus principais rivais na região a curto prazo.

Palavras-chave: União Europeia; Cáucaso; a Ásia Central; política externa; geopolítica.

Sumario. Introducción. 1. El Cáucaso meridional: progresos y frustraciones en el patio trasero de Bruselas. 1.1. De periferia soviética a foco de atención de Bruselas. 1.2. La Política Europea de Vecindad. Un salto cualitativo pero insuficiente. 1.3. La preeminencia del binomio seguridad-economía en la escala de prioridades de Bruselas. 2. Asia Central: impotencia en el "vecindario del vecindario" europeo. 2.1. Un recién llegado al "nuevo Gran Juego". 2.2. La renovada relevancia del Heartland en el tablero geopolítico mundial. 2.3. Un giro hacia la realpolitik en Asia Central. 3. Consideraciones finales. Adaptarse o morir en el corazón de Eurasia. Bibliografía.

Cómo citar: Jovaní Gil, Carles (2016) "La política exterior de la Unión Europea en el Cáucaso y Asia Central. Los límites de la apertura hacia el Este”. Geopolítica(s). Revista de estudios sobre espacio y poder, vol. 7, núm. 1, 109-129.

\section{Introducción}

En junio de 2015 Federica Mogherini, Alta Representante de la Unión Europea para Asuntos Exteriores y Política de Seguridad, anunció el propósito de presentar a mediados de 2016 la bautizada como Estrategia Global de la UE en Política Exterior y de Seguridad, que sustituirá la Estrategia Europea de Seguridad de 2003. Poco después, el informe Towards a New European Security Strategy? Assessing the Impact of Changes in the Global Security Environment, elaborado por la Dirección General para la Política Exterior del Parlamento Europeo, ponía de relieve la necesidad de reforzar la seguridad en el Cáucaso meridional así como de centrar la atención en las amenazas que emanaban de la región de Asia Central (Parlamento Europeo, 2015). La importancia de este territorio enclavado en las entrañas de Eurasia ha sido subrayada por importantes figuras como Zbigniew Brzezinski (1997: 123), quien se refirió a él como los "Balcanes euroasiáticos", o de manera más explícita por otros autores como Christopher Fettweis (2000: 58-71), quien llegó a afirmar que "[e]l Heartland ${ }^{3}$ de la masa continental euroasiática jugará un rol importante en el próximo siglo, y [...] tendrá una tremenda influencia sobre el carácter del sistema internacional”.

El influjo de la Unión Europea sobre el Cáucaso meridional y Asia Central fue meramente testimonial hasta bien entrada la década de los noventa. A medida que la integración comunitaria cristalizaba se hizo paulatinamente patente la necesidad de mirar hacia el Este no sólo como área propicia para futuras ampliaciones, sino también zona crítica para garantizar la estabilidad doméstica y, por ende, la sostenibilidad del proyecto político común. A lo largo de las últimas dos décadas la UE ha desarrollado una aproximación oficial de carácter eminente civil hacia la re-

3 En alusión al concepto acuñado por el geógrafo británico Halford Mackinder (1919) en su obra Democratic Ideals and Reality: A Study in the Politics of Reconstruction. Previamente, en "The Geographical Pivot of History”, se había referido a la misma región como “Área Pivote” (Mackinder, 1904). 
gión $^{4}$, con políticas destinadas —al menos sobre el papel— a reforzar su independencia y fomentar el desarrollo de la democracia y el respeto por los derechos humanos (Warkotsch, 2016: 1995). Sin embargo, no puede obviarse que Bruselas ha ejercido también un poder estructural y normativo (Manners, 2002) considerable sobre las esferas securitaria, económica e incluso psicológica de las respectivas repúblicas concernidas (Torbakov, 2013: 181).

La disonancia entre valores e intereses en el área de estudio se ha agudizado a la par que afloraban divergencias entre unos Estados miembros cada vez más imbuidos por la crisis que azota el continente de una lógica con tintes realistas (Klinke, 2012: 931). Si bien resulta desmedido proclamar la consagración de la geopolítica realista como base de la acción exterior de la UE, sí que puede apreciarse una creciente influencia de su sustrato sobre una praxis no siempre acorde con la retórica oficial ni las bondades pregonadas en los tratados fundamentales. Tal circunstancia justifica el recurso evocativo en las páginas que seguirán a la terminología propia de la disciplina. Realizadas estas precisiones, el presente artículo se propone estudiar la evolución de la política exterior de la UE en el área descrita durante la Posguerra Fría a fin de desvelar sus intereses y limitaciones, analizar las estrategias implementadas, y evaluar en qué medida ha sido capaz de socavar el domino de sus hegemones tradicionales.

\section{Mapa 1. Mapa político del Cáucaso meridional y Asia Central}

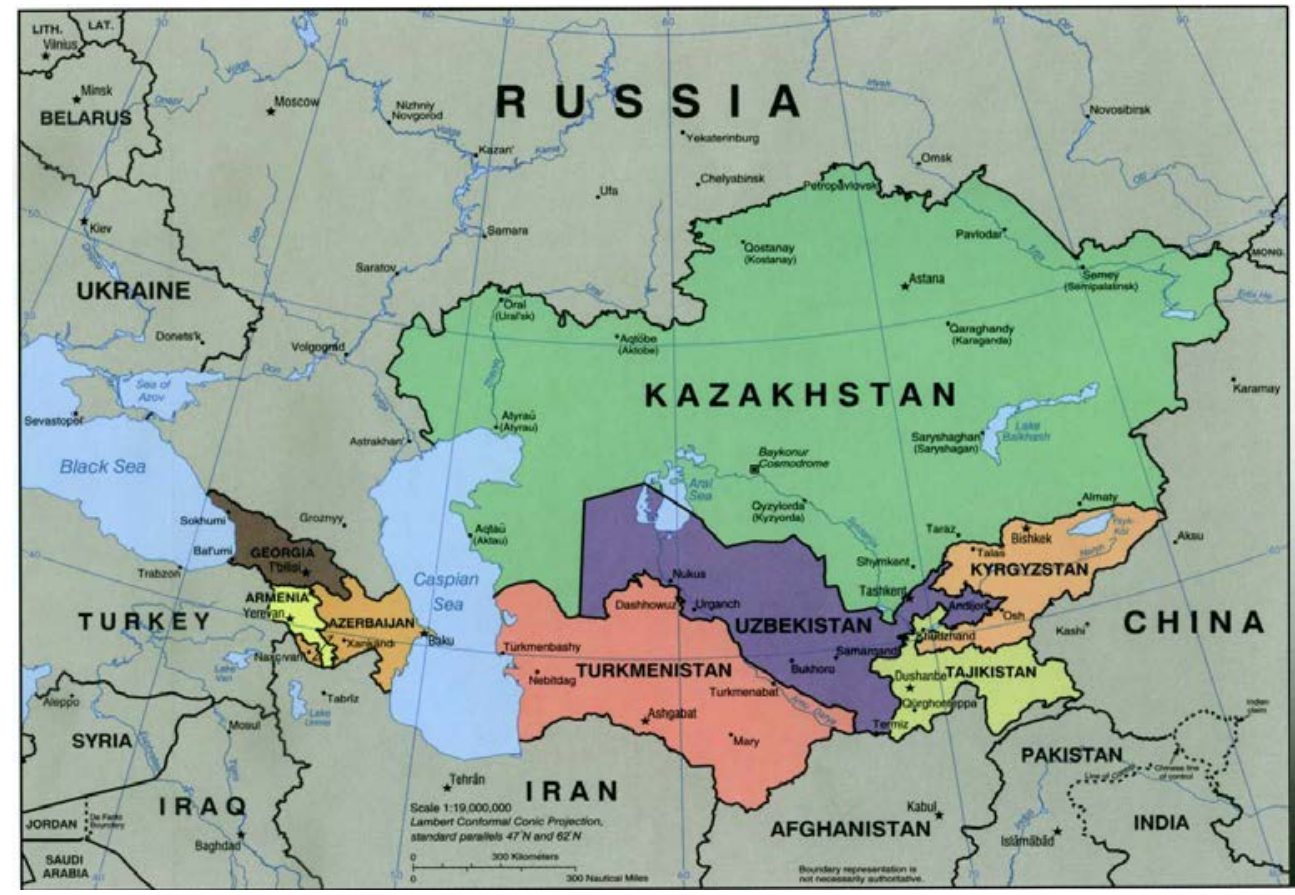

Fuente: The University of Texas at Austin (2016).

4 Véase a este respecto, el concepto de “Civilian Power” (Duchêne, 1972). 


\section{El Cáucaso meridional: progresos y frustraciones en el patio trasero de Bruselas}

El emplazamiento estratégico del Cáucaso meridional y su importancia como zona de producción y de paso de hidrocarburos desde la cuenca del Caspio a Occidente han atraído la atención de los arquitectos de la política exterior europea desde la desintegración de la URSS. Mirando al sur, la región limita con Turquía, miembro de la OTAN y eterno candidato al ingreso en la Unión Europea, así como con Irán, hostil hacia Occidente desde 1979 y foco de atención permanente debido a su controvertido programa de desarrollo nuclear y a los supuestos devaneos con el terrorismo internacional. Su frontera norte la custodia Rusia, potencia que se ha aprestado en los últimos tiempos a recuperar la tutela sobre sus antiguos vasallos soviéticos y que mira con recelo cualquier intento exógeno por ocupar el "vacío de poder" aparecido en 1991. A pesar de albergar unas reservas de hidrocarburos relativamente pequeñas, la inestabilidad del mercado internacional y la alta dependencia energética europea del gas ruso otorga al control de las infraestructuras energéticas del Cáucaso meridional una importancia sustantiva. Asimismo, su relativa proximidad a escenarios calientes como Irak, Siria o Afganistán lo dota de un valor único como puente logístico para las operaciones desarrolladas en Oriente Medio. A ello cabe sumar su abigarrada composición étnica y un cúmulo de tensiones secesionistas que han llevado a Nygren (2007: 101) a caracterizar a la región como el subcomplejo más inestable y volátil de cuantos componen el antiguo espacio soviético.

El análisis de las principales magnitudes que caracterizan la región y su comparación con las de la Unión Europea revelan que posee una población combinada inferior a la de Holanda, un PIB que apenas alcanza el $1 \%$ comunitario y un comercio que representa el $0,5 \%$ de las transacciones realizadas por Bruselas ${ }^{5}$. A pesar de la escasa envergadura de estas cifras, la UE ha expresado continuamente su interés en la zona por dos razones fundamentales: su cercanía y relativa disfuncionalidad; y la vulnerabilidad asociada al monopolio del suministro energético por parte de poderosos actores que operan a su vez en algunas de las zonas más inestables del planeta desde el punto de vista geopolítico (Comisión Europea, 2011).

Las amenazas más importantes a la seguridad comunitaria se derivan de la existencia en este territorio de diversos conflictos enquistados, de la operación en su seno de redes de delincuencia internacional y grupos fundamentalistas islámicos, así como del carácter autoritario de unos regímenes con capacidad para obstruir eventualmente el desarrollo de infraestructuras energéticas de interés para Bruselas como el gasoducto Transanatolio (TANAP) o el hasta la fecha postergado gasoducto Transcaspio. En la Estrategia Europea de Seguridad de 2003 se explicita la necesidad de "desarrollar un interés más fuerte y activo en los problemas del Cáucaso meridional, que a su debido tiempo será una región vecina” (Consejo de la Unión

No obstante, la UE sí que constituye un socio comercial prioritario para las tres repúblicas caucásicas. Según cifras de la Comisión Europea (2015a, b y c), el comercio con Bruselas representaba en 2014 el 28,8 \% de las exportaciones de Armenia, el 21,8 \% de las de Georgia y el 53,0 \% de las de Azerbaiyán. Resulta pertinente destacar que el peso de los hidrocarburos en las exportaciones era nulo en el caso armenio, mientras que alcanzaba el $20 \%$ en el caso georgiano (el 0,2 \% de las importaciones extracomunitarias) y el 98,8 \% en el azerí (el 2,9 \% de las importaciones extracomunitarias). 
Europea, 2003: 9). A los objetivos declarados de estabilidad, seguridad y desarrollo de la democracia y de las economías de mercado conviene añadir, no obstante, un último propósito más sibilino y al que raramente se alude de manera categórica en los documentos y declaraciones oficiales: la extensión de la influencia comunitaria hacia el Este a fin de desafiar y contrarrestar el peso tradicional de Rusia (Efe, 2012: 188-197).

\subsection{De periferia soviética a foco de atención de Bruselas}

La Declaración de Reconocimiento Mutuo entre la entonces Comunidad Europea y el COMECON firmada en 1989 abrió la puerta al establecimiento de relaciones entre Bruselas y las repúblicas soviéticas caucásicas, que en aquel momento todavía no gozaban de plena soberanía política. Tras la desintegración de la URSS la renombrada Unión Europea se involucraría paulatinamente en el antiguo espacio soviético a través del programa TACIS, cuyas prioridades en el Cáucaso meridional variarían en función del país afectado: mientras ponía énfasis en la reforma de la justicia en Georgia, redoblaba esfuerzos para la mejora del sector educativo así como la situación de la planta nuclear de Medzamor en Armenia, y abordaba la gestión de fronteras en Azerbaiyán. A pesar del voluntarismo que caracterizó estos esfuerzos iniciales, durante la primera mitad de los años noventa el papel de la UE en la seguridad del Cáucaso meridional fue prácticamente inexistente, de manera que sería la recién creada Federación Rusa quien capitalizara la gestión de los conflictos más importantes de la región (Simão, 2012: 15). A excepción de contribuciones puntuales de algunos de sus países miembros ${ }^{6}$, la UE se mostró reticente a la mediación en el avispero caucásico debido a diversos motivos: a los estragos causados por la crisis en los Balcanes, a la falta de una visión consensuada entre los socios europeos, a la inadecuación del marco legal, y al escaso desarrollo de instrumentos como la Política Exterior y de Seguridad Común (PESC), creada con el Tratado de Maastricht (Cornell y Starr, 2006: 11). La Comisión Europea se limitó a abrir en 1995 una única delegación en Tbilisi a la que se le encargaría la responsabilidad de gestionar la cooperación en materia humanitaria en las tres repúblicas surcaucásicas. Estos programas de asistencia privilegiaron como interlocutora a Rusia, que eclipsó en todo momento a Bruselas (Delcour, 2011: 3).

La Unión Europea impulsó en 1996 un nuevo marco contractual a través de los Acuerdos de Asociación y Cooperación (PCA). Se trataba de acuerdos mixtos dado que su contenido recaía en parte bajo la competencia directa de Bruselas y en parte bajo la de los Estados miembros. Su principal cometido era promover un clima adecuado para desarrollar el diálogo político, apoyar los esfuerzos de las nuevas repúblicas independientes para consolidar la democracia, completar la transición hacia una economía de mercado y estimular el desarrollo económico. Asimismo, pretendían también promover el comercio y la inversión además de la cooperación en materia legal, financiera, científico-tecnológica y cultural (Unión Europea, 2015). Los PCA firmados con Rusia, Ucrania y Moldavia incluían el objetivo adicional de crear las condiciones necesarias para el establecimiento de una futura

6 A modo de ejemplo, Italia, Suecia y Finlandia participaron en el Grupo de Minsk de la OSCE, creado para promover la resolución pacífica del conflicto de Nagorno Karabaj. 
área de libre comercio, un proyecto que no incluía a la región del Cáucaso meridional. En 1999 entraron en vigor los mencionados PCA en Armenia, Azerbaiyán y Georgia, con los que se alumbraría una plataforma para el diálogo en asuntos político-económicos y la promoción de la cooperación regional. Con todo, sus resultados iniciales fueron discretos debido a las dificultades de implantación de las reformas y a la incapacidad de la UE para atajar las causas últimas de la inestabilidad regional (Freire y Simão, 2008: 146).

En diciembre de 2000, el Parlamento Europeo solicitó con escaso éxito la creación de una "Dimensión Meridional" alrededor del mar Negro. Fueron acontecimientos como los atentados del 11-S, la Revolución de las Rosas de 2003, la ampliación de la UE o el enorme crecimiento económico de la región ${ }^{7}$ quienes acabarían determinando que Bruselas valorara su importancia estratégica en términos políticos, económicos y de seguridad. Así pues, en los años sucesivos se iniciarían una serie de movimientos aproximativos como el nombramiento en 2003 de Heikki Talvite como Representante Especial de la UE (REUE) para el Cáucaso meridional, o la alusión a la región en la Estrategia Europea de Seguridad presentada por Javier Solana en el Consejo Europeo de Tesalónica de 2003. El propio diplomático español, a la sazón Alto Representante de la Unión Europea para la Política Exterior y de Seguridad Común, viajó a Tbilisi en 2004 con un conjunto de propuestas de asistencia financiera a fin de apoyar los nuevos planes de reforma del gobierno. Asimismo, promovería también la puesta en marcha en julio de 2004 de la primera Misión de Estado de Derecho de la UE en Georgia, conocida como EUJUST Themis ${ }^{8}$ (Freire y Simão, 2008: 149-156).

\subsection{La Política Europea de Vecindad. Un salto cualitativo pero insuficiente}

El punto de partida de la Política Europea de Vecindad (PEV) fue la iniciativa propuesta en 2002 por el entonces presidente de la Comisión Romano Prodi en la comunicación Wider Europe-Neighbourhood: A New Framework for Relationship with Southern and Eastern Countries (Comisión Europea, 2003). Consolidada en julio de 2003, la PEV aspira a la difusión de los valores de la Unión, al mantenimiento de relaciones privilegiadas con los países vecinos, y a la adecuación de la acción exterior de la UE a las particularidades de cada Estado. Su principal objetivo es el de favorecer la cooperación en tres ámbitos esenciales: el securitario; el político, humano, científico y cultural; y el de desarrollo económico y social sostenible (Fernández, 2009: 10-11). La Comisión Europea, con el apoyo del Parlamento, recomendó la inclusión de las tres repúblicas caucásicas en la PEV, que se hizo efectiva en 2004. El mismo año se aprobó un documento estratégico para el diseño y la gestión de relaciones de carácter bilateral que serviría de base para la definición de los Planes de Acción. Éstos constituyen una herramienta política clave en la aproximación singularizada para cada país ${ }^{9}$, ligando necesidades y capacidades,

Las tres repúblicas surcaucásicas se situaban en 2006 en el top ten mundial en términos de crecimiento porcentual del PIB proyectado (Cornell y Starr, 2006: 14).

8 Diseñada con la finalidad de orientar la reforma de la justicia penal, apoyar las actividades anticorrupción y promover el respeto de los estándares internacionales y los derechos humanos.

9 Es ilustrativo el hecho de que mientras que el Plan de Acción de Armenia menciona el concepto de "autodeterminación de los pueblos”, el de Azerbaiyán alude al de "integridad territorial”. 
y definiendo simultáneamente objetivos en distintas áreas para un desarrollo sostenible, integrado y coherente (Freire y Simão, 2008: 147). Los planes de los tres Estados estudiados fueron aprobados en 2006, complementado así la cooperación establecida por los PCA vigentes. Sin embargo, dado que se trataba de documentos políticos sin rango de disposición legal, sus preceptos no eran de obligado cumplimiento, por lo que su efectividad y — por extensión— la de la PEV se ha visto en la práctica socavada.

En este sentido, los líderes armenios han manifestado en reiteradas ocasiones su intención de unirse a la Unión Europea en un futuro, quedando estipulado en la propia Estrategia Nacional de Seguridad que "el desarrollo y consolidación de las relaciones con las estructuras europeas, y con la Unión Europea en especial, es una prioridad para la política exterior del país” (Ministerio de AA. EE. de la República de Armenia, 2007). Ereván considera clave el acercamiento a Occidente para superar el embargo impuesto por Turquía y Azerbaiyán a principios de los noventa, pero todavía tiene por delante un largo recorrido en materia de mejora de los derechos políticos y libertades fundamentales. Asimismo, la no conclusión en septiembre de 2013 del Acuerdo de Asociación con la UE y el posterior ingreso en la Unión Económica Euroasiática (UEE) en 2015 han reducido significativamente las posibilidades de satisfacer sus aspiraciones europeas a medio plazo. Esta organización supraestatal aspira a la consolidación de un mercado único basado en la libertad de movimiento de personas, capitales, bienes y servicios. No obstante, los planes de Rusia no acaban ahí, sino que apuntan a una profundización de las relaciones políticas entre sus miembros (Van der Togt et al., 2015: 7).

Tal circunstancia justifica por qué la Unión Europea ha mostrado reservas hacia la nueva organización supranacional, más aún habida cuenta del delicado momento de su estrategia en el denominado "Vecindario Oriental". Existen dudas en Bruselas sobre los potenciales beneficios de un acercamiento, y no son pocos los analistas que han predicho el avance inexorable hacia un "Juego de Uniones" (Van der Togt et al., 2015: 8). En él, Moscú podría utilizar el nuevo bloque para contrapesar a la UE (Parlamento Europeo, 2016: 10). De hecho, Rusia y China se encuentran discutiendo la posibilidad de integrar a la UEE en el Silk Road Economic Belt impulsado desde Pekín (Pastukhova y Westphal, 2016: 7). El reconocimiento de la UEE como socio europeo podría además suponer la aceptación tácita de la hegemonía rusa sobre buena parte del antiguo espacio soviético, pero difícilmente disuadiaría a Moscú de sus apetencias sobre aquellos vecinos con claras incliaciones pro-europeístas como Ucrania o Georgia (Pozo, 2015: 9-10). En contrapartida, el cultivo de relaciones con la UEE podría también ofrecer algunas oportunidades. A modo de ilustración, "la UE podría negociar con Rusia en un espacio en el que su poder de negociación se vería limitado por el derecho a veto de los otros miembros” (Pozo, 2015: 8). Por consiguiente, una apuesta decidida por el multilateralismo en foros e instituciones internacionales combinada con una potente aproximación bilateral adaptada a cada uno de los Estados socios podría acabar minando la capacidad de liderazgo del Kremlin, obligando con ello a tejer amplios consensos entre ambos bloques.

Retomando la evaluación del alcance de la PEV, sus progresos han sido desiguales en el caso de Georgia. El Concepto Nacional de Seguridad de la república caucásica señala que el país "aspira a convertirse en parte de las estructuras euro- 
peas y euroatlánticas” (Ministerio de AA. EE. de la República de Georgia, 2011). El análisis de su situación interna revela mejoras significativas en materia de derechos humanos, lucha contra la corrupción y reformas económicas. Incluso se ha creado la figura ministerial para la Integración Euroatlántica. Avances como la conclusión de un Acuerdo de Asociación en junio de 2014 son importantes, aunque todavía insuficientes habida cuenta de la volubilidad política local. Por su parte, Azerbaiyán ha manifestado una actitud menos efusiva que sus dos vecinos surcaucásicos y tiene pocos incentivos para cooperar más allá del interés por equilibrar las relaciones entre Washington y Moscú. Aun así, en su Concepto Nacional de Seguridad figura la integración en las estructuras europeas y euroatlánticas como segunda mayor prioridad tras la restauración de la integridad territorial (Ministerio de AA. EE. de la República de Azerbaiyán, 2007). En esta línea, en noviembre de 2006 firmaría un Memorando de Entendimiento con la UE en el que se reconocía la asociación energética estratégica entre ambos actores (Efe, 2012: 197). A pesar de estos movimientos, los avances en materia de buena gobernanza y derechos humanos han sido tímidos, y el apoyo financiero occidental es también poco relevante al haberlo convertido su pujanza en donante neto de ayuda (Delcour y Duhot, 2011: 41).

Fernández (2009: 17) distingue cuatro obstáculos principales que han comprometido la efectividad de la PEV: las divergencias entre Estados miembros, la inadecuación de sus instrumentos, la dificultad para extender los valores europeos más allá de las fronteras comunitarias, y la gestión de los conflictos congelados en el antiguo espacio soviético. Además, en el seno de la UE existen discrepancias crecientes respecto a las líneas directrices de la política exterior común. Éstas se manifiestan particularmente en relación a la política hacia Rusia, país que incide directamente en las relaciones tanto con las repúblicas surcaucásicas como con las centroasiáticas. Mientras que la postura de los líderes bálticos y de la Europa oriental ha sido tradicionalmente hostil a Moscú, Francia y Alemania prefieren preservar sus buenas relaciones con el gigante eslavo debido a la fuerte dependencia energética. Otra de las manzanas de la discordia son los instrumentos requeridos para la puesta en marcha de la política exterior y, en particular, el requisito de "condicionalidad” ${ }^{10}$ democrática exigido por Bruselas. Asimismo, se ha criticado también la actitud de la UE por su enfoque excesivamente técnico y la escasa interacción con las sociedades concernidas (Boonstra y Delcour, 2015: 5). En última instancia, los logros en la promoción de los valores comunitarios tampoco han estado exentos de ambivalencia debido en gran medida a "una serie de preferencias divergentes, ambigüedades y desfases institucionales en la actividad de los Estados miembros" (Fernández, 2009: 18-19).

\subsection{La preeminencia del binomio seguridad-economía en la escala de priori- dades de Bruselas}

Tal y como aparece en la Estrategia de Seguridad Europea y en los documentos clave de la PEV, la prevención de conflictos constituye un elemento medular de la

10 En el caso del proceso de ampliación de la Unión Europea, el requisito de condicionalidad se basa en el cumplimiento de los criterios de Copenhague y del acervo comunitario. 
política exterior comunitaria en su vecindario. La Unión Europea fue hasta la guerra de agosto de 2008 el principal donante de ayuda en Abjasia y Osetia del Sur, si bien apenas ha interferido en la resolución del contencioso de Nagorno Karabaj, que en la primavera de 2016 vivió de nuevo una escalada de la violencia. Ejemplos como la puesta en marcha del Instrumento de Estabilidad ${ }^{11}$ o el nombramiento de un REUE para el Cáucaso meridional atestiguan la creciente importancia que otorga Bruselas a esta cuestión. Uno de los mayores éxitos de esta nueva figura institucional fue el de impulsar una política de involucramiento con las entidades secesionistas de Georgia destinada a acabar con el tradicional monopolio de la intermediación rusa.

En la misma línea, la mediación de la presidencia francesa de la Unión fue crucial para facilitar un acuerdo de alto el fuego en la guerra de Georgia de 2008. Con posterioridad al fin de la actividad armada, se procedería al despliegue de la Misión de Observación de la Unión Europea para Georgia (EUMM) y al establecimiento de la Misión Independiente Internacional de Verificación de Hechos sobre el Conflicto de Georgia (IIFFMCG) ${ }^{12}$, medidas ambas que pusieron de relieve la rápida capacidad de actuación en términos de decisión, financiación y despliegue. Sin embargo, el impulso que representó la guerra para la credibilidad de la UE como actor securitario internacional tendría un corto recorrido. Relegada al estatus de observador en Osetia del Sur y a facilitar apoyo para la aprobación de medidas de generación de confianza en Abjasia ${ }^{13}$, el rol de la UE en la gestión de conflictos ha sido marginal (Whitman y Wolff, 2010: 96) a pesar del amplio abanico de instrumentos políticos que tiene a su alcance ${ }^{14}$. El alcance de la PEV a este respecto se ha visto limitado por las dificultades a la circulación en las entidades separatistas, la falta de medios para implementar medidas de generación de confianza y seguridad, y el diseño propio de una política de vecindad basada legalmente en acuerdos bilaterales y carente de una visión regional de conjunto (Delcour y Duhot, 2011: 13-14; Simão, 2012: 16-19).

Otras iniciativas regionales lanzadas bajo el paraguas de la PEV muestran debilidades similares. La Sinergia del Mar Negro ${ }^{15}$ fue aprobada el 15 de abril de 2007 con el propósito de conferir valor añadido a cuestiones transfronterizas que no podían tratarse a nivel bilateral. Sus pobres resultados reflejan, no obstante, las dificultades para abordarse de manera multilateral cuestiones de seguridad en la región (Delcour, 2011: 10). La Unión Europea inauguró en mayo de 2009 la Asociación

11 El Instrumento de Estabilidad es una herramienta política y financiera a disposición de la Unión Europea cuyos objetivos consisten en dar respuesta a necesidades urgentes vinculadas a situaciones de inestabilidad política o desastre mayor; generar condiciones que permitan garantizar la seguridad política y el desarrollo económico a largo plazo; y participar en la gestión de crisis a través del apoyo a las operaciones de la Política de Seguridad y Defensa Común.

12 El objetivo de esta misión, aprobada por el Consejo el 2 de diciembre de 2008, no era otro que investigar los orígenes y el curso del conflicto desde el punto de vista del derecho internacional y humanitario.

13 Rusia concluyó en enero de 2015 un Tratado sobre la Alianza y Asociación Estratégica con Abjasia, y en marzo de 2015 hizo lo propio con un Tratado sobre la Alianza e Integración con Osetia del Sur. Con ello atraía todavía más a ambas entidades secesionistas a la órbita de Moscú.

14 Entre ellos se encuentran las Declaraciones Conjuntas; las Acciones Conjuntas; las Estrategias Comunes; las Posiciones Comunes; los Representantes Especiales; las sanciones económicas; las operaciones civiles, policiales y políticas; el apoyo a la sociedad civil, y los proyectos de democratización.

15 Forman parte de esta iniciativa Grecia, Turquía, Bulgaria, Rumanía, Moldavia, Ucrania, Rusia, Georgia, Armenia y Azerbaiyán. 
Oriental, una iniciativa que proporciona una aproximación dual track al combinar las relaciones bilaterales tradicionales con el cultivo del multilateralismo (Amaro, 2013: 261). Integrada por Bielorrusia, Moldavia, Ucrania, Armenia, Georgia y Azerbaiyán, la Asociación Oriental pone énfasis en cuatro dimensiones políticas: relaciones bilaterales, multilaterales, gobernanza y asistencia financiera. En el ámbito de las relaciones bilaterales prevé Acuerdos de Asociación que deben sustituir progresivamente a los PCA, mientras que a nivel multilateral se centra en proyectos energéticos (Mikhelidze, 2009: 2). A pesar de su potencial, no hace mención alguna a las entidades no reconocidas en la región, por lo que su impacto en la estabilización política de las mismas ha sido prácticamente nulo.

Bruselas reformó en enero de 2007 su estructura de financiación externa al reemplazar MEDA, TACIS y otros programas con el Instrumento Europeo de Vecindad y Asociación, que asiste la implementación de la PEV y cuenta en el período 2014-2020 con un presupuesto de 15.400 millones de euros, destinados mayoritariamente a la financiación de las reformas contenidas en los Planes de Acción nacionales (Babayan, 2010). La UE había ofreció también a Georgia (2005), Armenia y Azerbaiyán (2009) el General System of Preferences (GSP+), un paso transicional hacia la apertura total del mercado europeo cuya implementación estaría condicionada a la ratificación e implementación de 27 convenciones en materia de derechos humanos, laborales y buena gobernanza (Pardo, 2011: 236). En el marco de la Asociación Oriental, los Deep and Comprehensive Free Trade Agreements (DCFTA) han incorporado provisiones para el establecimiento de un área de libre circulación de bienes, servicios, capitales e inversión.

El lento incremento de la influencia económica de la UE sobre la región ha sido el resultado de una serie de dinámicas que se detallan a continuación. En primer lugar, Bruselas no emprendió hasta después de agosto de 2008 una evaluación en profundidad de los pronósticos para el establecimiento de DCFTA. La guerra supuso un espaldarazo para los abogados de una aproximación holística al Cáucaso meridional, lo que se tradujo en un reforzamiento de la dimensión oriental de la PEV a través de la Asociación Oriental. En segundo lugar, la participación comercial de la UE no parece haber sido suficiente para su integración en al área económica comunitaria. En Armenia, el poder se encuentra todavía ligado a grupos próximos al actual presidente Serzh Sargsián, quedando por consiguiente poco espacio para la transformación del paradigma político-económico. Por lo que respecta a Azerbaiyán, la práctica totalidad de las exportaciones a la UE están relacionadas con el gas y el petróleo, por lo que el requisito de condicionalidad es frecuentemente obviado por Bruselas y los incentivos para asumir los costes de un DCFTA son escasos (Pardo, 2011: 238-239).

El acuerdo institucional primario e integral que une la región del mar Negro y la Unión Europea es el Tratado sobre la Carta de la Energía de 1997, que pretendía crear un mercado energético abierto y diversificado en el que también participara Rusia. El Consejo Europeo de marzo de 2009 entronizó como eje energético prioritario al "corredor meridional”, un proyecto que incluye los gasoductos Transadriático (TAP), Turquía-Grecia-Italia (ITGI), Transcaspio y Nabucco (Efe, 2012: 196). En el caso de este último, la falta de entendimiento entre los principales actores afectados fue aprovechada por Rusia para conseguir un contrato de explotación exclusiva del campo de gas azerí de Shah Deniz, una de las fuentes que debían 
alimentarlo. Ello, unido a la propuesta del TANAP, acabó por aparcar el propio proyecto Nabucco, obligando por consiguiente a Bruselas a reformular el diseño mismo de la infraestructura de transporte energético hacia Europa occidental. Esta circunstancia ilustra cómo pese a que la Unión Europea es vista como un club que puede crear prosperidad y estabilidad, su idiosincrasia y la complejidad de la región confieren a Rusia una ventaja comparativa esencial a la hora de seguir preservando su condición de polo de referencia en el Cáucaso meridional.

\section{Asia Central: impotencia en el "vecindario del vecindario" europeo}

La localización estratégica de Asia Central ha determinado que infinidad de civilizaciones la hayan transitado y ocupado a lo largo de los siglos. De hecho, su delimitación actual como unidad de estudio diferenciada se debe en gran medida a los avatares de la historia y, muy en particular, a la ingeniería étnica soviética. La vaporosidad del término nos obliga a circunscribirlo en el presente estudio a las cinco repúblicas independientes nacidas en la ribera oriental del mar Caspio tras la desintegración de la URSS. Sus aproximadamente cincuenta millones de habitantes, la mayoría de los cuales de habla turcófona -a excepción de los tayikos-, se organizan en sociedades clánicas de confesión suní aunque fuertemente secularizadas. Al igual que el Cáucaso meridional, las particularidades geográficas de esta región han determinado que venga siendo objeto de deseo de las principales potencias desde el seísmo de 1991. La riqueza en hidrocarburos, la cercanía de Oriente Medio y Afganistán, su valor como zona de paso de importantes infraestructuras energéticas y rutas comerciales, la proliferación del islamismo radical, las tensiones fronterizas, el separatismo y la inestabilidad crónica han acabado convirtiéndola en el escenario contemporáneo de una férrea pugna por la hegemonía en el corazón del continente euroasiático.

La lejanía y el elemento disuasorio que representaba la férula soviética resultó en un escaso interés histórico de la Unión Europea por la región. Sin embargo, la progresiva ampliación de las fronteras comunitarias hacia el Este ha acercado progresivamente a las cinco repúblicas centroasiáticas a Bruselas hasta consagrarlas como nueva "segunda periferia" continental. Esta relocalización ha sido favorecida por el creciente interés de la UE en reivindicar una esfera de influencia propia en la escena global, por la necesidad de reducir la dependencia energética de Rusia, y también por los desafíos críticos para su seguridad que emanan de este territorio. En los últimos años, la retórica europea del cultivo de la buena gobernanza y la defensa acerada de los derechos humanos ha sido relegada a un segundo plano en aras de una potencial mejora de las relaciones con los regímenes locales. Estas renuncias son sintomáticas, no obstante, de la debilidad comunitaria, y no bastan para poder disipar las reservas de los líderes locales.

\subsection{Un recién llegado al "nuevo Gran Juego”}

El reconocimiento oficial en 1988 entre la Comunidad Europea y el COMECON puede considerarse como el primer antecedente al establecimiento de relaciones formales entre Bruselas y las repúblicas centroasiáticas. A principios de los años 
noventa sólo Alemania contaba con embajadas en los cinco Estados nacidos de los rescoldos de la URSS, mientras que la mayoría de países europeos disponía únicamente de representantes ubicados regularmente en Moscú o Ankara. La primera delegación comunitaria en Asia Central abrió sus puertas en Kazajistán en 1994, lo cual es indicativo del escaso grado de desarrollo de los vínculos bilaterales y explica en gran medida la exclusión de las repúblicas concernidas de instituciones como el Consejo de Europa ${ }^{16}$. Estas circunstancias llevan a De Jong y Wouters (2011: 7) a calificar la aproximación inicial de la entonces Comunidad Europea a la región como "ad hoc, azarosa y plagada de incertidumbres".

La cooperación económica con Asia Central se inició en 1991 a través del programa TACIS, centrado en un primer momento en materia energética y de seguridad nuclear y, con posterioridad, también en la reestructuración de empresas estatales, el desarrollo del sector privado y la reforma de la administración pública. TACIS fue inicialmente inconsistente debido a la inestabilidad de la región y a la falta de interés de la propia Unión Europea. Con el paso del tiempo, llegaría a gestionar una plétora de programas creados por el Plan de Acción de la Comisión Europea, e implementados de manera conjunta con el Programa de las Naciones Unidas para el Desarrollo (PNUD), la Oficina de las Naciones Unidas para la Droga y la Criminalidad, la OSCE y la Organización Internacional de las Migraciones.

Entre ellos, el Proyecto Interestatal de Petróleo y Gas a Europa (INOGATE), creado en 1995, contribuyó a que las nuevas repúblicas independientes consiguieran la gestión autónoma de las infraestructuras energéticas heredadas del período soviético, a su modernización y a la orientación de su gestión hacia el mercado exterior de acuerdo con las normas y estándares internacionales. La política energética recibiría un nuevo impulso en noviembre de 2004 con la Iniciativa de Bakú, que reunió a Estados clave del mar Negro, el mar Caspio y el Cáucaso con el propósito de establecer unos parámetros de armonización de los estándares legales y técnicos, avanzar en la integración de los sistemas energéticos, modernizar las infraestructuras y promocionar el desarrollo de nuevos proyectos (Denison, 2009: 5). El Corredor de Transporte Europa-Cáucaso-Asia (TRACECA), operativo desde 1993, incluía las ocho repúblicas centroasiáticas y caucásicas en un proyecto que pretendía conectar Europa Occidental con el Lejano Oriente. Debido a su importancia, fue transformado en 2000 en una comisión intergubernamental reconocida por la ONU.

Los límites operativos de TACIS residían en el carácter condicionado de la ayuda así como en los déficit cognitivos derivados de la concepción de Asia Central como una región unitaria. De ahí que tras su extinción fuera reemplazado en 2007 por el Instrumento de Cooperación para el Desarrollo (DCI), con un presupuesto de 1.072 millones de dólares para Asia Central en el período 2014-2020 ${ }^{1 f}$. La otra gran herramienta de la UE para aproximarse a las nuevos Estados independientes, los Acuerdos de Asociación y Cooperación (PCA), tiene un carácter bilateral y no está sujeta al estricto cumplimiento del dictado de Bruselas. Los PCA cuentan con

16 No es ése el caso actual de las repúblicas surcaucásicas. Georgia se incorporó en abril de 1999, y Armenia y Azerbaiyán harían lo propio en enero de 2001.

17 Pese a la aparente magnitud de la cifra, los fondos destinados a Asia Central son relativamente pequeños en relación a los consignados a otras regiones del antiguo espacio soviético. Compárense con el presupuesto de 15.433 millones de euros del Instrumento Europeo de Vecindad para el período 2014-2020. 
una base jurídica más ambigua y buscan la conformación de "un espacio de diálogo y concertación política, una liberalización con el objetivo de aumentar los intercambios comerciales, y un refuerzo de las nuevas líneas de cooperación acordadas por los socios” (Rocha, 2012: 750). Entre estas últimas destaca el control sobre el armamento y la vigilancia de los movimientos islamistas locales ${ }^{18}$. Los primeros países en negociar un PCA fueron Kazajistán ${ }^{19}$, Uzbekistán y Kirguistán, con respectivos acuerdos concluidos en 1996 y vigentes desde 1999. En el caso de Tayikistán, la guerra civil pospuso el cierre de las negociaciones hasta 2009, mientras que la preocupación por la situación de los derechos humanos en Turkmenistán ha postergado hasta el momento la ratificación del texto firmado en 1998. En su lugar se encuentra vigente desde 2008 un Acuerdo Comercial Interino que suple parcialmente sus funciones (De Jong y Wouters, 2011: 10).

\subsection{La renovada relevancia del Heartland en el tablero geopolítico mundial}

Los atentados terroristas del 11-S y la subsiguiente campaña militar en Afganistán pusieron de relieve una vez más el interés estratégico de la región. Este cambio de paradigma en las relaciones internacionales llevó a Bruselas a poner a punto el Programa de Cooperación Regional de Asia Central, diseñado para el período 2002-2006. El documento incidía en la necesidad de mejorar los procesos democráticos y el respeto a los derechos humanos en una región que tras la puesta en marcha de la Política de Europea de Vecindad —en la que no se incluían las repúblicas centroasiáticas - pasaba a constituir una segunda periferia de la UE (Rocha, 2012: 758). El creciente interés por parte de la Unión Europea se hizo de nuevo patente en 2003 con la apertura de una delegación comunitaria en Dusambé, a la que seguiría un año más tarde la de una Casa de Europa en Taskent ${ }^{20}$. En julio de 2005 se iniciaría un nuevo capítulo de relaciones con el nombramiento del primer REUE para Asia Central, cuyo mandato primordial debía ser la promoción del cambio democrático. Su actitud crítica durante hechos de Andiján, que llegaron a suponer la suspensión parcial del PCA uzbeko ${ }^{21}$, no fue óbice para que Bruselas siguiera considerando la región de interés desde el punto de vista geoestratégico. A modo de ilustración, el Green Paper de marzo de 2006 reconocía la importancia de la seguridad energética tras las crisis del gas de Ucrania ${ }^{22}$ (Comisión Europea, 2006),

18 Una de las facciones fundamentalistas islámicas con mayor proyección es la del Partido de la Liberación Islámica. Fundado en los años cincuenta en Jordania, desde donde se extendió a todo el mundo, se encuentra dirigido desde 2003 por Ata Abu Rashta y persigue la creación de una gran nación político-religiosa ( $M a-$ warah-al-nahr) en Asia Central. Numerosos integrantes de esta y otras organizaciones análogas han viajado a Siria para combatir junto al Estado Islámico (Boonstra, 2015: 2).

19 El 21 de diciembre de 2015, Kazajistán firmó un Acuerdo de Asociación y Cooperación Mejorado, convirtiéndose en la primera república centroasiática en hacerlo.

20 En la actualidad, la UE posee delegaciones en todas las repúblicas centroasiáticas a excepción de Turkmenistán, en la que previsiblemente se abrirá una en un futuro próximo (Gast, 2014).

21 La suspensión se prolongó hasta octubre de 2009, e incluía tres tipos de sanciones: una prohibición de visados contra doce oficiales del gobierno de Taskent, el embargo a las exportaciones de armas europeas, y el cese de relaciones bilaterales a alto nivel. Desde el levantamiento de estas medidas, las relaciones han mejorado ostensiblemente. A modo de ejemplo, en enero de 2011 se firmó el Acuerdo sobre el Establecimiento de una Oficina de Representación Diplomática en Taskent, y en febrero de 2012 se lanzó un Proyecto para la Asistencia a la Reforma del Sistema Judicial y Legal uzbeko.

22 De acuerdo con el BP Statistical Review of World Energy de 2014 (British Petroleum, 2014), Kazajistán posee las mayores reservas de petróleo de la región con 30.000 millones de barriles (el 1,8 \% mundial), seguido de 
abogando incluso por la creación de una macrorregión energética sobre las líneas del Tratado Comunitario de la Energía (ENCOM) ${ }^{23}$.

La presidencia alemana del Consejo durante la primera mitad de 2007 declaró a Asia Central como área prioritaria y se propuso trabajar en una nueva estrategia para la región. En esta línea, el Consejo Europeo aprobaría el verano del mismo año dos documentos estratégicos: el Regional Strategy Paper for Assistance to Central Asia for the Period 2007-2013 y The European Union and Central Asia: The New Partnership in Action. El segundo suponía en esencia la toma de conciencia de la insuficiencia de la actuación de la UE en la región hasta ese momento, e identificaba siete áreas de abordaje prioritario: derechos humanos ${ }^{24}$, imperio de la ley, democratización, buena gobernanza, medio ambiente, lucha contra amenazas comunes y diálogo intercultural (Consejo de la Unión Europea, 2007). El texto adquirió el carácter propio de un documento marco que debía permitir a los Estados miembros desarrollar acciones bilaterales así como a Bruselas diseñar proyectos y medidas concretas en función de las necesidades de cada país y de los resultados de la aplicación de los PCA (Fernández, 2009: 28). La principal innovación que incorporaba era la celebración de encuentros multilaterales entre las cinco repúblicas centroasiáticas y la troika europea ${ }^{25}$, incluido un Diálogo de Derechos Humanos con encuentros anuales a nivel oficial (Emerson y Boonstra, 2010: 65).

En el ámbito energético, la estrategia apuntaba a la dependencia de Bruselas y la necesidad de inversión en la región como elementos centrales de las relaciones bilaterales. Sobre la base de los objetivos establecidos en la Iniciativa de Bakú, la UE pretendía mejorar la seguridad energética a través de la convergencia de los mercados energéticos; la diversificación y el ajuste a la demanda; la transparencia y la generación de confianza; la mejora de la eficiencia en la gestión; la atracción de inversiones; la rehabilitación de infraestructuras, y la ampliación de la red (Consejo de la Unión Europea, 2007: 23-24). La guerra de Georgia de 2008 obligaría a Bruselas a presentar un Second Strategic Energy Review (SSER2) en el que se hacía hincapié en la necesidad de diversificar las fuentes, de diseñar mecanismos de respuesta ante la crisis, de desarrollar el "corredor meridional” y de explorar un posible mecanismo de compra en bloque de gas del Caspio a través de la creación de la Caspian Development Corportation (CDC) (Consejo de la Unión Europea, 2014). El mismo año, la Comisión Europea y Turkmenistán firmarían un Memorando de Entendimiento que preveía el suministro de diez mil millones de metros cúbicos de

Uzbekistán y Turkmenistán con 600 millones de barriles respectivamente. En cuanto a las reservas de gas, Turkmenistán ocupa la primera posición con 17,5 billones de metros cúbicos (el 9,4 \% mundial), seguido de Kazajistán con 1,5 billones de metros cúbicos (el 0,8 \% mundial) y Uzbekistán con 1,1 billones de metros cúbicos (0,6 \% mundial). Según cifras de la Comisión Europea (2015d), el comercio con las cinco repúblicas centroasiáticas apenas representó en 2014 el 1,0 \% del total de la UE. En concreto, el 91,8 \% de las importaciones procedentes de esta región pertenecían a hidrocarburos y derivados.

23 Firmado el 25 de octubre de 2005 y en vigor desde el 1 de julio de 2006, dio lugar al establecimiento de una comunidad de estados en la que las partes se comprometían a implementar el acervo comunitario, a desarrollar un marco regulatorio adecuado y a liberalizar el mercado energético.

24 Esta área se reforzaría con la creación a finales de 2006 del Instrumento Europeo para la Democracia y los Derechos Humanos (EIDHR).

25 Con anterioridad a la entrada en vigor del Tratado de Lisboa en 2009, el término troika aludía al triunvirato europeo en política exterior constituido por el ministro de Asuntos Exteriores del estado que ostentaba la presidencia del Consejo, el Alto Representante para la Política Exterior y de Seguridad Común, y el comisario europeo encargado de las relaciones exteriores y la política de vecindad. 
gas a Europa occidental a través del gasoducto Nabucco, así como también el desarrollo de otras infraestructuras energéticas en fase de estudio como el White Stream $^{26}$ y el ya reseñado gasoducto Transcaspio (Emerson y Boonstra, 2010: 7780).

En el ámbito de la seguridad, la Unión Europea estima crucial —al menos a nivel declaratorio - el mantenimiento de una estrecha colaboración con las repúblicas centroasiáticas. Si bien no dispone de una estrategia de Reforma del Sector de la Seguridad $(\mathrm{SSR})^{27}$, Bruselas se ha convertido en uno de los donantes internacionales más importantes a través de fondos de la Comisión y de iniciativas como la European Police Mission en Afganistán de 2007. El principal vínculo entre el apoyo europeo a la SSR en Afganistán y en Asia Central ha sido el control de fronteras, financiado en gran medida por la UE e implementado por el PNUD (Boonstra, 2011: 2-3). Las críticas más comunes vertidas a esta estrategia han apuntado precisamente a su excesiva concentración tanto en la asistencia a la coalición occidental en la campaña afgana como en el acceso a los recursos energéticos, relegando a un segundo plano la promoción a largo plazo de la estabilidad, el desarrollo de la sociedad civil, el cumplimiento de la ley y las reformas democráticas. Su lenidad en esta materia hace que el concepto de buena gobernanza haya quedado reducido en opinión de Rocha (2012: 762-763) a una mera “dimensión instrumental”, limitándose a la promoción de reformas administrativas y eludiendo cambios estructurales de gran calado. Esta aproximación, calificada también de "pragmatismo forzado" por el mismo autor (Rocha, 2012: 762-763) y de simple realpolitik por parte de Graubner (2008), salió a relucir en los episodios de inestabilidad que sacudieron Uzbekistán (2005), Kirguistán (2005 y 2010), Kazajistán (2001) y Tayikistán (2010 y 2012). Bruselas adoptó en todos ellos una postura discreta, limitándose a emitir comunicados genéricos de denuncia ante las violaciones de los derechos humanos (Rocha, 2012: 763).

\subsection{Un giro hacia la realpolitik en Asia Central}

A partir del verano de 2012, la Unión Europea inició un paulatino distanciamiento de su aproximación tradicional basada en el "poder blando" y centrada en la promoción de la democracia, los derechos humanos y el respeto de la Ley. La revisión por parte de los ministros de Asuntos Exteriores de la estrategia aprobada cinco años atrás marcó un punto de inflexión en el que las cuestiones de "seguridad dura" pasaron a ocupar un primer plano. Tal cambio puede explicarse por los recelos ante la retirada de la coalición occidental de Afganistán previsto para finales de 2014, por los avances de Rusia hacia el establecimiento de la Unión Económica Euroasiática $^{28}$, así como por la progresiva toma de conciencia de la necesidad de desarrollar

26 Se trata de un gasoducto submarino con una capacidad anual de 32.000 millones de metros cúbicos anuales que conectaría Georgia y Rumanía a través del lecho del mar Negro.

27 La SSR hace referencia al proceso de reforma del sector de la seguridad de un estado. Responde a una situación en la que la inoperancia en materia securitaria disminuye la capacidad de la propia Administración para garantizar la seguridad tanto de sus instituciones como de su población.

28 El tratado que daba carta de naturaleza a su creación fue firmado el 29 de mayo de 2014, y entró en vigor el 1 de enero de 2015. Actualmente la integran Armenia, Bielorrusia, Kazajistán, Kirguistán y Rusia. 
un papel más activo en un mundo en el que la Vieja Europa ha perdido peso relativo (Gast, 2014).

El renovado interés por cuestiones de seguridad quedó reflejado en el Progress Report on the Implementation of the EU Strategy for Central Asia de 2012 (Servicio Europeo de Acción Exterior y Comisión Europea, 2012), donde se confirmaba el establecimiento de un Diálogo de Seguridad a Alto Nivel UE-Asia Central ${ }^{29}$. Posteriormente, las Council Conclusions on the EU Strategy for Central Asia de 2015 (Consejo de la Unión Europea, 2015) pondrían de nuevo énfasis en el refuerzo de la cooperación en operaciones contraterroristas y de vigilancia fronteriza. Pese a que algunos de los objetivos contenidos en ambos documentos se han llevado a la práctica con aparente éxito, como por ejemplo la celebración de un primer Diálogo de Seguridad en Bruselas en junio de 2013, la retórica voluntarista comunitaria ha chocado sistemáticamente con la escasez real de medios para poder contrarrestar de manera efectiva la influencia de potencias con mucho mayor peso en la región como Rusia, Estados Unidos o China.

\section{Consideraciones finales. Adaptarse o morir en el corazón de Eurasia}

La Unión Europea ha ampliado su actividad en el Cáucaso meridional fundamentalmente a través de canales económicos, si bien subrayando también las ventajas que pueden derivarse de la conclusión de acuerdos políticos. Apoyar a estos países en su proceso de transición no ha sido un desafío fácil habida cuenta de sus dificultades domésticas en materia de gobernanza, de la inestabilidad políticoinstitucional y de los desequilibrios económicos y sociales. En este contexto, la UE se ha consagrado como un actor relevante en la facilitación de asistencia financiera, técnica y humanitaria, así como en la promoción de la resolución de la conflictividad local (Freire y Simão, 2008: 164). La aproximación de Bruselas a la región a través de un marco institucionalizado cooperativo ha reforzado asimismo la generación de confianza además de mejorar la imagen de la UE en las sociedades afectadas (Pardo, 2011: 241).

Sin embargo, factores como la débil institucionalización, el elevado grado de corrupción, el desarrollo insuficiente, la presencia de conflictos latentes y los pobres avances en materia democratización, desarrollo del Estado de Derecho y derechos humanos comprometen el objetivo estratégico de la PESC de extender la zona de seguridad en la periferia suroriental del continente europeo (Fernández, 2009: 5). De la misma manera, en un entramado institucional que concentra la mayoría de decisiones en materia de seguridad en el voto unánime del Consejo, resulta muy difícil alcanzar posiciones comunes en casos donde los intereses nacionales se encuentran involucrados. Esta realidad ha llevado a Ditrych (2011: 3) a comparar a la UE con la policefalia de una Hidra. Todo ello, unido a la ausencia de herramientas de "seguridad dura", al rechazo al recurso a la condicionalidad negativa y a la falta de una política consensuada en áreas clave como la energía, nos lleva a concluir que la influencia comunitaria en el Cáucaso meridional, pese a haber experimenta-

29 Sin embargo, los resultados de las conferencias de 2013 y 2015, las únicas celebradas hasta el momento, se saldaron con progresos modestos debido, en gran medida, al escaso entusiasmo con el que fueron tratadas por parte de las repúblicas centroasiáticas (Boonstra, 2015: 2). 
do una progresión ascendente desde la desintegración soviética, se ve todavía limitada por una serie de factores estructurales que categorizan a Bruselas como un actor subalterno a la sombra de Moscú.

En cuanto a la política exterior de la Unión Europea en Asia Central, la adopción de una nueva estrategia en 2007 supuso en la práctica el abandono del institucionalismo liberal propio de la tradición comunitaria en pro de una aproximación pragmática destinada a conseguir progresos concretos. Con la perspectiva que ofrece el paso del tiempo, resulta de justicia reconocer que este documento ha permitido elevar el perfil de Bruselas en la región, por lo que no es previsible su revisión a corto plazo. Existe mayor conocimiento y comprensión de la realidad sobre el terreno, se han producido tímidos avances en materia de derechos humanos, y se han visto beneficiadas campañas como la afgana. Sin embargo, la crisis económica que azota el continente europeo y los conflictos de Ucrania, Siria o contra el Estado Islámico siguen ilustrando la posición subsidiaria que todavía ocupa este territorio en la escala de prioridades comunitarias (Boonstra, 2011: 6).

A pesar de las sucesivas evaluaciones positivas oficiales, la realidad es que la Unión Europea sigue siendo en Asia Central un actor marginal que carece siquiera de una estrategia integral con objetivos realistas y medios para alcanzarlos. La profundización de las relaciones bilaterales no se ha traducido en una mejora significativa de los estándares democráticos locales, lo cual no es de extrañar habida cuenta de la escasa importancia concedida a este aspecto en los últimos años. Tampoco se han materializado en la medida de los esperado las anheladas contrapartidas en materia económica. Turkmenistán no ha llegado a comprometerse al establecimiento de volúmenes de gas para el corredor energético meridional (Latypov, 2015), y sigue sin resolverse la construcción del gasoducto Transcaspio.

El área que configuran el Cáucaso meridional y Asia Central se encuentra íntimamente ligada a las dinámicas de seguridad que sostienen el nuevo orden emergente en una franja que se extiende desde el Caspio al Lejano Oriente. En un escenario de creciente competitividad, sus propias repúblicas han abierto puntualmente las puertas a Bruselas a fin de contrarrestar el dominio tradicional de Moscú, el influjo político omnímodo de Washington y el amenazante poderío económico chino. Proyectos como la Unión Económica Euroasiática plantean nuevos desafíos para una Unión Europea inmersa en una crisis existencial que ha llevado al límite sus costuras y la ha despojado de su hábito liberal. Los males crónicos que socavan sus cimientos, los recelos entre los nuevos Estados independientes y la oposición contumaz de sus principales rivales comprometen cualquier aspiración comunitaria de consagrarse a medio plazo como un actor preeminente en este espacio (Melvin, 2012: 5). Queda por ver si la futura Estrategia Global de la UE en Política Exterior y de Seguridad será capaz de integrar una aproximación mejor adaptada a la singularidad de la región, así como a las necesidades del momento histórico a fin de conciliar con mayor eficiencia el imperativo categórico fundacional y la realpolitik. 


\section{Bibliografía}

Amaro, V. (2013) "The EU and Russia: Competing Discourses, Practices and Interests in the Shared Neighbourhood". Perspectives on European Politics and Society, vol. 14, núm. 2, 256-271.

Babayan, N. (2010) “Fear Or Love Thy Neighbour? EU Framework of Fostering”. Journal of Contemporary European Research, vol. 1, 40-56.

Boonstra, J. (2011) The EU's Interests in Central Asia: Integrating Energy, Security and Values into Coherent Policy. Bonn: EDC 2020.

Boonstra, J. (2015) Reviewing the EU's Approach to Central Asia. Policy Brief núm. 34, Madrid: FRIDE.

Boonstra, J. y Delcour, L. (2005) Una región rota: Análisis de las políticas de la UE en el sur del Cáucaso. Policy Brief núm. 119, Madrid: FRIDE.

British Petroleum (2014). BP Statistical Review of World Energy.

Brzezinski, Z. (1997) The Grand Chessboard. American Primacy and its Geostrategic Imperatives. Nueva York: Basic Books.

Comisión Europea (2003) Wider Europe-Neighbourhood: A New Framework for Relationship with Southern and Eastern Countries. COM 104, Bruselas.

Comisión Europea (2011) Energy Roadmap 2050. Impact Assessment Part 1 Including Part I of Annex 1. SEC(2011)1565/1, Bruselas.

Comisión Europea, Dirección General para el Comercio (2015a) "European Union, Trade in goods with Armenia” [URL: <http://trade.ec.europa.eu/doclib/docs/2006/september/ tradoc_113345.pdf $>$. Consultado el 11 de diciembre de 2015].

Comisión Europea, Dirección General para el Comercio (2015b) “European Union, Trade in goods with Azerbaijan” [URL: <http://trade.ec.europa.eu/doclib/docs/2006/ september/tradoc_113383.pdf>. Consultado el 11 de diciembre de 2015].

Comisión Europea, Dirección General para el Comercio (2015c) European Union, Trade in goods with Georgia” [URL: <http://trade.ec.europa.eu/doclib/docs/2006/ september/tradoc_113347.pdf.pdf>. Consultado el 11 de diciembre de 2015].

Comisión Europea, Dirección General para el Comercio (2015). "European Union, Trade in goods with Central Asia” [URL: <http://trade.ec.europa.eu/doclib/docs/2013/ november/tradoc_151896.pdf $>$. Consultado el 7 de abril de 2016].

Consejo de la Unión Europea (2003). Estrategia Europea de Seguridad. Una Europa segura en un mundo mejor. Bruselas.

Consejo de la Unión Europea (2007) The European Union and Central Asia: The New Partnership in Action. Bruselas.

Consejo de la Unión Europea (2008) Second Strategic Energy Review. Bruselas.

Consejo de la Unión Europea, Consejo de Asuntos Exteriores (2015) “Council Conclusions on the EU Strategy for Central Asia”, 10191/15 [Puesto en línea el 22 de junio de 2015. URL: < http://data.consilium.europa.eu/doc/document/ST-10191-2015-INIT/es/pdf>. Consultado el 18 de noviembre de 2015].

Cornell, S. E., y Starr, F. (2006) The Caucasus: A Challenge for Europe. Washington D.C.: Central Asia-Caucasus Institute \& Silk Road Studies Program.

Damro, C. (2012) “Market Power Union”. Journal of European Public Policy, vol. 19, núm. 5, 682-699.

De Jong, S., y Wouters, J. (2011) Central Asia and the EU's Drive Towards Energy Diversification. Lovaina: Leuven Centre for Global Governance Studies. 
Delcour, L. (2011) “The European Union’s Policy in the South Caucasus: In Search of a Strategy”, en A. Jafalian (ed.) Reassessing Security in the South Caucasus. Farnham: Ashgate, 177-194.

Delcour, L., y Duhot, H. (2011) Bringing South Caucasus Closer to Europe: Achievements and Challenges in ENP Implementation. Natolin: College of Europe.

Denison, M. (2009) The EU and Central Asia: Commercialising the Energy Relations. Bruselas: EU-Central Asia Monitoring.

Ditrych, O. (2011) EU Security Policy in the South Caucasus. the Need to Move from Hydra to Hercules. Berlín: German Institute for International and Security Affairs.

Duchêne, F. (1972) “Europe’s Role in World Peace”, en R. Mayne (ed.) Europe Tomorrow: Sixteen Europeans Look Ahead. Londres: Fontana, 32-47.

Efe, H. (2012) "Foreign Policy of the European Union Towards the South Caucasus". International Journal of Business and Social Science, vol. 3, núm. 17, 187-199.

Emerson, M., y Boonstra, J. (2010) Into EurAsia: Monitoring the EU's Central Asia Strategy. Bruselas-Madrid: CEPS y FRIDE.

Fernández, N. (2009) Asia Central y el Cáucaso Sur en la estrategia de la UE. Documento de trabajo 21/2009. Madrid: Observatorio Asia Central - Real Instituto Elcano.

Fettweis, C. (2000) "Sir Halford Mackinder, Geopolitics and Policy Making in the 21st Century”. Parameters. U.S. Army War College Quarterly, vol. 30, núm. 2, 58-71.

Freire, M. R., y Simão, L. (2008) “A política europeia de vizinhança para o Cáucaso do Sul: Interesses competitivos e oportunidades de cooperação”. Nação e Defesa, vol. 119, núm. 4, 141-166.

Gast, A. S. (2014) A Shift in the EU Strategy for Central Asia? Moscú: Carnegie Moscow Center.

Graubner, C. (2008) “EU Strategy on Central Asia: Realpolitik After All”. Central AsiaCaucasus Analyst, 14 de mayo.

Klinke, I. (2012) "Postmodern Geopolitics? The European Union Eyes Russia”. EuropeAsia Studies, vol. 64, núm. 5, 929-947.

Latypov, E. (2015) "Fully-fledged Southern Gas Corridor by 2020: European Gas Race between Iran and Turkmenistan”, CEEP [Puesto en línea el 24 de octubre de 2015. URL: <http://www.ceep.be/southern-gas-corridor-european-gas-iran-turkmenistan>. Consultado el 14 de diciembre de 2015].

Mackinder, H. J. (1904) “The Geographical Pivot of History”. The Geographical Journal, vol. 23, núm. 4, 421-437.

Mackinder, H. J. (1919) Democratic Ideals and Reality: A Study in the Politics of Reconstruction. Londres: Constable and Company.

Manners, I. (2002) “Normative Power Europe: A Contradiction in Terms?”. Journal of Common Market Studies, vol. 40, núm. 2, 235-258.

Melvin, N., y Prelz, G. (2015) Managing Conflict and Integration in the South Caucasus: A Challenge for the European Union. SIPRI-CASCADE Policy Brief, 31 de octubre, SIPRI-CASCADE.

Mikhelidze, N. (2009) Eastern Partnership and Conflicts in the South Caucasus: Old Wine in New Skins? Roma: Istituto Affari Internazionali.

Ministerio de Asuntos Exteriores de la República de Armenia (2007) Estrategia Nacional de Seguridad de la República de Armenia [URL: <http://www.mfa.am/u_files/ file/doctrine/Doctrineeng.pdf $>$. Consultado el 12 de diciembre de 2015]. 
Ministerio de Asuntos Exteriores de la República de Azerbaiyán (2007) Concepto Nacional de Seguridad de la República de Azerbaiyán [URL: <http://www.azembassy.org.au/ uploads/docs/Azerbaijan.pdf>. Consultado el 3 de diciembre de 2015].

Ministerio de Asuntos Exteriores de la República de Georgia (2011) Concepto Nacional de Seguridad de Georgia [URL: <http://www.mfa.gov.ge/index.php?lang_id =ENG\&sec_id=12>. Consultado el el 14 de dicembre de 2015].

Nygren, B. (2007) The Rebuilding of Greater Russia: Putin's Foreign Policy Towards the CIS Countries. Abingdon: Routledge.

Pardo, O. B. (2011) “No man's Land? A Comparative Analysis of the EU and Russia's Influence in the Southern Caucasus”. Communist and Post-Communist Studies, vol. 44, 233-243.

Parlamento Europeo, Dirección General para la Política Exterior (2015) Towards a New European Security Strategy? Assessing the Impact of Changes in the Global Security Environment. PE 534.989, Bruselas.

Parlamento Europeo, Dirección General para la Política Exterior (2016) The EU in Central Asia: The Regional Context. PE 535.020, Bruselas.

Pastukhova, M., y Westphal, K. (2016) A Common Energy Market in the Eurasian Economic Union. SWP Comment, febrero. Berlín: German Institute for International and Security Affairs.

Pozo, G. (2015) The Eurasian Economic Union: Ambitions and Vulnerabilities of the 'other EU', ARI 49/2015, 8 de octubre, Madrid: Real Instituto Elcano.

Rocha, M. (2012) "La política exterior de la Unión Europea en Asia Central: De la condicionalidad política al pragmatismo”. Anuario Mexicano de Derecho Internacional, vol. XII, 731-777.

Servicio Europeo de Acción Exterior y Comisión Europea (2012) "Progress Report on the Implementation of the EU Strategy for Central Asia”, 11455/2012 [URL: $<$ http://eeas.europa.eu/central_asia/docs/20120628_progress_report_en.pdf >. Consultado el 16 de diciembre de 2015].

Simão, L. (2012) "EU-Russia Security Relations: Lessons from the South Caucasus”, en R. E. Kanet y M. R. Freire (eds.) Competing for Influence: The UE and Russia in PosSoviet Eurasia. Dordrecht: Republic of Letters, 157-178.

The University of Texas at Austin (2016) “Caucasus and Central Asia (Political) 2003”. Perry-Castañeda Library Map Collection [Puesto en línea el 1 de abril de 2016. URL: $<$ http://www.lib.utexas.edu/maps/commonwealth/caucasus_cntrl_asia_pol_00.jpg>. Consultado el 2 de abril de 2016].

Torbakov, I. (2013) "The European Union, Russia and the «In-between Europe“, en T. Cierco (ed.) The European Union Neighbourhood: Challenges and Opportunities. Londres: Routledge, 173-190.

Unión Europea (2010) "Partnership and Cooperation Agreements (PCAs): Russia, Eastern Europe, the Southern Caucasus and Central Asia” [Puesto en línea el 29 de septiembre de 2010. URL: <http://europa.eu/legislation_summaries/external_relations/ relations_with_third_countries/eastern_europe_and_central_asia/r17002_en.htm>. Consultado el 25 de noviembre de 2015].

Van der Togt, T.; Montesano, F., y Kozak, I. (2015) From Competition to Compatibility. Striking a Eurasian Balance in EU-Russia Relations. La Haya: Netherlands Institute of International Relations Clingendael. 
Warkotsch, A. (2016) “The Caspian Sea Region: The Struggle for Resources”, en S. Gstöhl y E. Lannon (eds.) The Neighbours of the European Union's Neighbours. Londres: Routledge, 181-202.

Whitman, R. G., y Wolff, S. (2010) "The EU as a Conflict Manager? The Case of Georgia and its Implications”. International Affairs, vol. 86, núm. 1, 87-107. 